\title{
Chemical composition and antifungal activity of the volatile oil from Epichlö̈ gansuensis, endophyte-infected and non-infected Achnatherum inebrians
}

\author{
ZHANG XingXu* ${ }^{*}$ XIA Chao, LI ChunJie \& NAN ZhiBiao* \\ State Key Laboratory of Grassland Agro-Ecosystems, College of Pastoral Agriculture Science and Technology, Lanzhou University, Lanzhou \\ 730020, China
}

Received December 31, 2014; accepted January 7, 2015; published online March 31, 2015

Citation: Zhang XX, Xia C, Li CJ, Nan ZB. Chemical composition and antifungal activity of the volatile oil from Epichloë gansuensis, endophyte-infected and non-infected Achnatherum inebrians. Sci China Life Sci, 2015, 58: 512-514, doi: 10.1007/s11427-015-4837-0

Endophytic fungi that belong to the genus Epichlö (including species previously classified in the genus Neotyphodium) have been found in many cool-season grasses (subfamily Pooideae) [1]. Achnatherum inebrians (Hance) Keng (drunken horse grass, DHG) is a toxic perennial bunchgrass, distributed mainly throughout the harsh conditions of alpine or sub-alpine grasslands in the northwest of China. This species is usually infected by the fungal endophyte Epichloë gansuensis [1,2], which apparently enhances its resistance to the abiotic and biotic stress [3].

The dried above-ground plant samples (100 g) of endophyte-infected (E+) or endophyte-free (E-) DHG and dried mycelia of E. gansuensis were ground to a powder, so as to pass through a 1-mm sieve, and then subjected to hydro-distillation, with the samples steam distilled for $3 \mathrm{~h}$ using a Clevenger-type collector. The resulting hydrodistillate of samples were extracted through ethyl acetate to obtain the oil. The oils were dried over anhydrous sodium sulphate $\left(\mathrm{Na}_{2} \mathrm{SO}_{4}\right)$, then rotary evaporated, leaving a concentrate of the essential oil (a faint yellow color). The essential oil was stored in $-20^{\circ} \mathrm{C}$ refrigerator under $\mathrm{N}_{2}$ in a sealed vial until required. Gas chromatography-mass spectrometry (GC-MS) analysis was carried out to determine the composition of the essential oil from A. inebrians and $E$. gansuensis. Identification of the compounds was performed by the retention indices (RI), and authentic reference com-

*Corresponding author (email: zhibiao@1zu.edu.cn) pounds, peak matching library searches, as well as a database of the National Institute of Standards and Technology (NIST). Identification of the oil compounds is expressed as percent peak area relative to total peak area from the MS analyses of the whole extracts [4].

The volatile oil yield of E+ A. inebrians was $0.11 \%(\mathrm{~g} / \mathrm{g}$ fresh weight) by hydro-distillation. Sixty-two compounds were identified in the E+ plants. The main components were heptadecane $(9.06 \%) ; 2^{\prime}, 3^{\prime}, 4^{\prime}$-trimethoxyacetophenone (7.82\%); octadecane $(5.95 \%)$; tetracontane $(5.63 \%)$; octadecane, 3-ethyl-5-(2-ethylbutyl) (4.95\%), etc. The volatile oil yield of $\mathrm{E}-A$. inebrians was $0.09 \%$ (g/g fresh weight) by hydro-distillation. Sixty compounds were identified in the E- plants. The main components were heptadecane (10.28\%); pentadecane, 2,6,10-trimethyl (7.17\%); terthexadecanethiol $(5.59 \%)$; hexadecane $(5.72 \%)$ and 1,2benzenedicarboxylic acid, bis-(2-methylpropyl) ester $(5.12 \%)$. There were 31 compounds identified in both E+ and $\mathrm{E}$ - plants. Of these, the main components were heptadecane; hexadecane; octadecane; 3-ethyl-5-(2-ethylbutyl); heptacosane and tert-hexadecanethiol. The content of pentadecane; tetradecane, 2,6,10-trimethyl; hexadecanoic acid, methyl ester; tetratetracontane; octadecane, 3-ethyl-5-(2ethylbutyl) and ethyl iso-allocholate were greater in the E+ plants. The content of 17-pentatriacontene; nonadecane, and pentadecane, 2,6,10-trime-thyl were greater in the E- plants. The volatile oil yield of E. gansuensis was $0.09 \%$ (g/g fresh weight) by hydro-distillation. There were 34 compounds 
identified in the E. gansuensis culture solution. The main components were 1,3-dioxolane $(75.63 \%)$; 1-hexacosene $(1.83 \%)$ and eicosane $(1.54 \%)$. Seven compounds were shown as common to both the E+ and E- plants and to $E$. gansuensis.

Inhibitory activity of the DHG volatile oil on mycelial growth of the fungal pathogens was measured according to the method of Zhang et al. [5]. Alternaria alternata and Bipolaris sorokiniana were isolated from A. inebrians. Curvularia lunata, Fusarium avenaceum, Fusarium solani and Trichoderma viride were isolated from Poa pratensis. Colonies of A. alternata, B. sorokiniana, and C. lunata were inhibited significantly $(P<0.05)$ by $\mathrm{E}+($ vs. E-) volatiles under an oil concentration from 0.02 to $2 \mathrm{mg} \mathrm{mL}^{-1}$ and showed increased inhibition with increasing oil dose, while colonies growth of A. alternata, B. sorokiniana, and $C$. lunata were stimulated at $0.01 \mathrm{mg} \mathrm{mL}^{-1}$ concentration. $F$. avenaceum, $F$. solani, and $T$. viride also showed some degree of growth inhibition by the volatile oils, the difference between the respective $\mathrm{E}+$ and $\mathrm{E}$ - oil treatments being significant $(P<0.05)$ except at the lowest concentration of 0.01 $\mathrm{mg} \mathrm{mL}^{-1}$ (Figure 1).

Although the presence of E. gansuensis apparently affected some qualitative and quantitative differences in the production of DHG volatile compounds, this oil should also be more fully studied for possible important associations within DHG. For example, what may be the implications for DHG oil components in regulating the growth of E. gan-
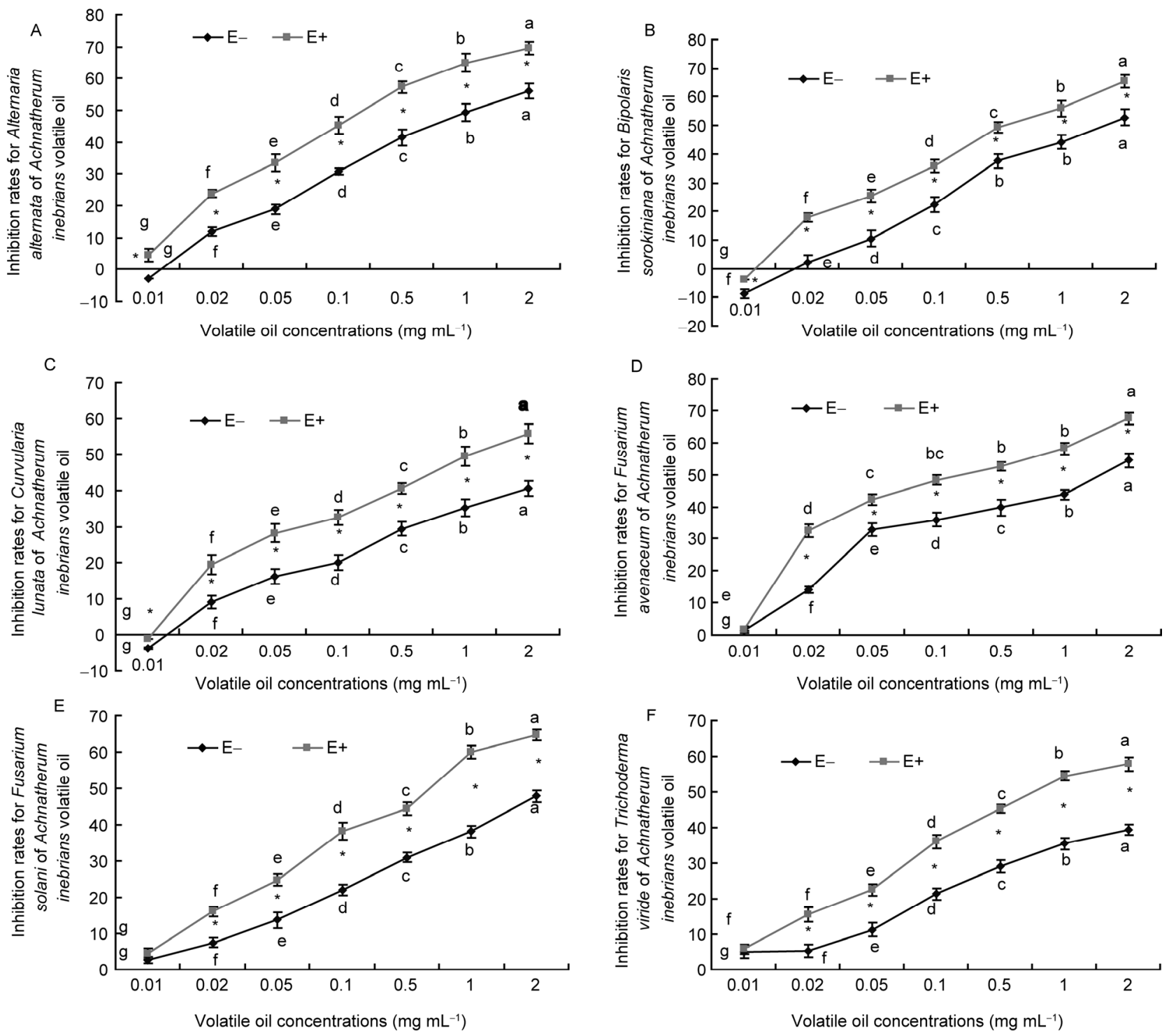

Figure 1 Inhibition rates for 6 different fungi using (E+/E-) Achnatherum inebrians volatile oils. Each treatment was for all three samples ( $n=3$ ). Values are mean \pm standard error (SE), with bars indicating SE. Columns with non-matching letters indicate a significant difference at $P<0.05$ (Duncan test). The asterisk (*) indicates significant difference $(P<0.05)$ between $\mathrm{E}+$ and $\mathrm{E}$ - volatile oil treatments at the same concentrations. 
suensis to prevent mutualism from becoming parasitism? The specific volatile compounds that affect fungal pathogens and the antifungal mechanisms of DHG volatile oil also need to be further clarified.

We thank Prof. Peter Long and Dr. Corry Matthew for polishing the English and giving beneficial discussion of the experiment, Qi WeiYan, Zhang ZhiXin, Li Ya and Liu TianZeng for their help and advice during the preliminary stages of this project. This work was supported by the National Basic Research Program of China (2014CB138702), the National Natural Science Foundation of China (31402132), the Fundamental Research Funds for the Central Universities (lzujbky-2014-75) and the Program for Changjiang Scholars and Innovative Research Team in University (IRT13019).
1 Leuchtmann A, Bacon, C W, Schardl C L, White J F, Tadych M. Nomenclatural realignment of Neotyphodium species with genus Epichlö̈. Mycologia, 2014, 106: 202-215

2 Li C J, Nan Z B, Paul V H, Dapprich P, Liu Y. A new Neotyphodium species symbiotic with drunken horse grass (Achnatherum inebrians) in China. Mycotaxon, 2004, 90: 141-147

3 Zhang X X, Nan Z B, Li C J, Gao K. Cytotoxic effect of ergot alkaloids in Achnatherum inebrians infected by the Neotyphodium gansuense endophyte. J Agr Food Chem, 2014a, 62: 7419-7422

4 Kordali S, Aslan I, Calmasur O, Cakir A. Toxicity of essential oils isolated from three Artemisia species and some of their major components to granary weevil, Sitophilus granaries (L.) (Coleoptera: Curculionidae). Ind Crops Prod, 2006, 23: 162-170

5 Zhang XX, Wu YP, Nan ZB. Antifungal activity of petroleum ether extracts from Achnatherum inebrians infected with Neotyphodium gansuense. Sci China Life Sci, 2014b, 57: 1234-1235

Open Access This article is distributed under the terms of the Creative Commons Attribution License which permits any use, distribution, and reproduction in any medium, provided the original author(s) and source are credited. 